\section{Pharmacologic Management of Overactive Bladder and Urge Incontinence: A Review of New and Old Treatment Options}

\section{Introduction}

Overactive bladder, particularly when accompanied by urinary incontinence, is a medical condition that affects a significant number of patients and drastically impacts quality of life. In the United States alone, the prevalence of overactive bladder with or without urge incontinence is $16.0 \%$ in men and $16.9 \%$ in women [1]. Unfortunately, many of the current mainstays of therapy come with side effects and interactions that are especially challenging in the patient population in which prevalence of incontinence is highest. Drugs with alternative mechanisms of action have recently been under development, providing well-tolerated options for longterm management. Here, we will review the most commonly used medications to treat overactive bladder, as well as newer options that have become available.

\section{Classification of Urinary Dysfunction}

The term urinary incontinence is broad, and encompasses several subtypes of urinary tract dysfunction with different etiologies. Stress incontinence is due to loss of urethral support, most often secondary to pelvic floor muscle weakening. Urge incontinence is due to inappropriate contraction of the detrusor muscle in the bladder and is one of the major criteria in diagnosing overactive bladder syndrome. In women, the most common types of urinary incontinence are stress incontinence and urge incontinence. Some women may have symptoms of both, which is called "mixed urinary incontinence." In men, the most common mechanism by which urinary incontinence occurs is from urethral constriction by an enlarged prostate. Both benign prostatic hyperplasia and prostate cancer lead to increased prostate size and impingement on normal urethral flow, resulting in urge incontinence [2]. Urge incontinence may or may not give the patient a sensation of the need to urinate prior to leakage of urine from the urethra.

\section{Urinary Tract Anatomy and Physiology}

To fully understand the mechanisms of both urinary incontinence and its pharmacologic management, it is important to have a clear foundation of the anatomy and physiology of the urinary system, especially the bladder.

The bladder wall consists of several layers, each with a different cell type and function. The innermost layer is composed of transitional epithelium. Below this layer is a network of blood vessels and a layer of smooth muscle fibers called the detrusor muscle. The detrusor is the primary muscle responsible for bladder contractility, and its function is critical to maintaining continence [3].

\section{Journal of}

\section{Andrology \& Gynaecology}

Leslie Matthews, Robert Shapiro * and Stanley

Zaslau

Department of Obstetrics and Gynecology, West Virginia University School of Medicine, USA

*Address for Correspondence

Dr. Robert Shapiro, MD, Assistant Professor, Department of Obstetrics and Gynecology, West Virginia University School of Medicine, USA; E-mail: rshapiro@wvuhealthcare.com

Submission: 18 February 2014

Accepted: 01 April 2014

Published: 03 April 2014

During micturition, the detrusor muscle in the wall of the bladder contracts and increases intra-vesical pressure. Eventually the pressure inside the bladder exceeds the pressure in the outflow tract, and urine is permitted to flow out of the bladder and through the relaxed external urinary sphincter [4].

Within the wall of the bladder is a rich network of parasympathetic efferent fibers and postganglionic cell bodies. There is little sympathetic innervation of the bladder; therefore the primary neurotransmitter involved in voluntary micturition is acetylcholine. In the male urinary tract, the neck of the bladder does contain sympathetic innervation to prevent retrograde ejaculation, thus responds to adrenergic stimulation as well $[3,4]$. The afferent nerve fibers in the bladder are activated by increased intra-vesical pressure, bladder stretch, as well as existing urine flow. These fibers initiate signals to urinate when the bladder is full, as well as to continue urinating until the bladder is empty [3].

Maintaining continence requires coordination between autonomic nervous system regulation and muscle activity in the bladder. Disruptions in the normal activity of either can result in the release of urine at inappropriate times and a great deal of distress to the patient [2]. Medications used to treat urge urinary incontinence and overactive bladder generally target the autonomic nervous system and detrusor smooth muscle to reduce unnecessary contraction and leakage of urine.

\section{Anticholinergic Medications}

The mainstays of treatment for urge incontinence and overactive bladder are anticholinergic, or antimuscarinic, agents. Currently in the United States, there are six anticholinergics approved for the treatment of urge urinary incontinence in both men and women. All work by inhibiting the binding of acetylcholine to muscarinic receptors in the bladder smooth muscle. Of the five muscarinic receptors, the detrusor contains primarily M2 and M3 subtypes [5]. Both M2 and M3 activation by acetylcholine initiate G-protein coupled receptor transduction cascades in the bladder [6]. Therefore, inhibition of neurotransmitter binding will decrease the activity of the signaling pathways causing the unwanted detrusor contractions leading to urine release. However, nonselective anticholinergic agents 
Citation: Matthews L, Shapiro R, Zaslau S. Pharmacologic Management of Overactive Bladder and Urge Incontinence: A Review of New and Old Treatment Options. J Androl Gynaecol. 2014;2(1): 4.

ISSN: 2332-3442

will also bind to muscarinic receptors systemically, producing many side effects in several locations.

In general, all anticholinergics in this group have the potential to exert the same adverse effects, which are mediated by the inhibition of acetylcholine binding to muscarinic receptors throughout the body. These side effects include cognitive dysfunction, dry eye, dry mouth, sedation, and constipation. These can be quite bothersome to patients, enough so that discontinuation has proven to be a challenge in long-term use of these medications. Therefore, it is important to consider which medications have selective receptor binding, or alternative dosage forms that may minimize adverse effects. It is also important to use the lowest effective dose so as to maintain efficacy while minimizing toxicity [6].

The prototype agent, oxybutynin, was approved in 1975 as an immediate release formulation, which is still currently available. An advantage to immediate release oxybutynin is the ability to titrate dosing based on treatment goals and individual response to therapy. Also, immediate release preparations are widely covered by insurance plans making their access affordable. However, immediate release oxybutynin has the highest incidence of adverse effects and the highest rate of discontinuation of all the anticholinergics in its class [7]. In particular, oxybutynin crosses the blood brain barrier and may cause cognitive impairment especially in the elderly [8]. Dosing multiple times daily may also be complicated for some patients who struggle with adherence. For ease of dosing and minimization of side effects, an extended release formulation was developed and approved in 1999. The active drug is contained in an insoluble shell, and is slowly released through a small pore over a 24 -hour period. Since the shell is insoluble, it is excreted unchanged in the feces. Therefore, it is important to remind patients they may see remnants of the shell in their stool, which is completely normal and harmless [9]. Oxybutynin is also the only anticholinergic drug for overactive bladder that is available as a transdermal delivery system as well. Transdermal oxybutynin is now available for over-the-counter use. Transdermal administration prevents first-pass metabolism and hepatic conversion to the active metabolite, $\mathrm{N}$-desethyloxybutynin, which is implicated in the majority of systemic side effects of the drug. The transdermal system also delivers a steady, low concentration of drug, further minimizing side effects and toxicities. Better tolerability and twiceweekly dosing make transdermal oxybutynin an attractive option for patients who may struggle with adherence to therapy. Patients may experience local irritation with the transdermal formulation, but applying the patch to a different site with each administration can minimizes sensitivity to patch components [10].

\section{Oxybutynin}

- Key PointsImmediate release formulation allows for dose titration to response

- Side effects limit adherence

- Extended release and transdermal formulations provide ease of dosing and minimize side effects

- Transdermal formulation is available over-the-counter

- Use with caution in the elderly as it may cause impaired cognition or exacerbate pre-existing dementia.
In 1998, tolterodine became the next anticholinergic agent approved for urge incontinence. Extended release tolterodine was approved in 2000, offering once daily dosing as opposed to the twice daily dosing recommended for the immediate release product [11]. Tolterodine was found in preclinical studies to have greater specificity for the muscarinic receptors in the bladder versus those in the parotid gland, indicating a potential decrease in systemic side effects as compared to oxybutynin. It also has a low lipid solubility which makes it less likely to cross the blood brain barrier. Therefore, it should have minimal, if any propensity to cause cognitive impairment. A 2001 meta-analysis demonstrated similar efficacy data between tolterodine and oxybutynin, but a statistically significant reduction in side effects in the tolterodine group. These patients also had fewer discontinuations due to toxicities as compared to those patients taking oxybutynin [12]. Development of this drug was evidence that muscarinic receptor selectivity was an important factor contributing to the severity of side effects and rates of adherence.

Tolterodine: Key Points

- Similar efficacy to oxybutynin

- Fewer side effects due to greater selectivity for bladder receptors

- Extended release formulation allows for once-daily dosing

- Less likely to cause cognitive impairment in the eldery

Trospium was approved by the Food and Drug Administration (FDA) in 2004, and is less commonly used than the other anticholinergics in its class. When compared to the efficacy of oxybutynin immediate release, many studies have demonstrated non inferiority. However, several studies, including one large multicenter study in Germany showed a significant reduction in side effects such as dry mouth in the trospium group [13]. Trospium also has very poor central nervous system penetration, minimizing cognitive side effects as compared to other anticholinergics [14]. Additionally, trospium is not metabolized by CYP450 enzymes in the liver, which could be advantageous for patients at risk for significant drug interactions [15]. Unfortunately, there have not been many studies comparing trospium to the other newer anticholinergics, so a clear role in therapy has yet to be determined.

Trospium: Key Points

- Clinical efficacy versus other anticholinergics is not well defined

- Lower incidence of side effects, particularly cognitive effects

- Fewer drug interactions due to non-CYP metabolism

Darifenacin selectively inhibits the M3 receptor in the detrusor muscle, with very little M2 inhibition. The FDA approved darifenacin in 2004. Given its high selectivity for the M3 receptor, darifenacin maintains efficacy but has significant reductions in side effects compared to nonselective anticholinergics, such as oxybutynin. In particular, it has a low incidence of cognitive side effects, which would be beneficial especially in elderly patients [16]. Darifenacin is a substrate of several hepatic CYP enzymes, including the 3A4 and 2D6 isoforms. Therefore, the potential for interactions with CYP inducers 
Citation: Matthews L, Shapiro R, Zaslau S. Pharmacologic Management of Overactive Bladder and Urge Incontinence: A Review of New and Old Treatment Options. J Androl Gynaecol. 2014;2(1): 4.

or inhibitors should be considered when using darifenacin [17].

Darifenacin: Key Points

- Similar efficacy to other anticholinergic agents

- Highly selective for the M3 receptor in the bladder

- $\quad$ Low incidence of cognitive side effects

- $\quad$ Potential for interactions with CYP inhibitors and inducers

Solifenacin was approved in 2005, and has demonstrated improved efficacy and tolerability over tolterodine extended release. Patients reported both reduction in incontinence episodes as well as improvements in quality of life [18]. Like darifenacin, solifenacin also is a CYP450 substrate, thus has the potential for interactions with inducers and inhibitors of the P450 system, particularly the 3A4 isoform [17]. A pharmaco-economic evaluation in 2006 also determined that solifenacin had the greatest cost-effectiveness when compared to all other anticholinergics, including extendedrelease and transdermal formulations. The study examined clinical outcomes as well as direct drug costs and medical costs associated with overactive bladder-induced comorbidities [19]. This could be an advantage in terms of overall economic burden and may be a consideration in formulary management.

Solifenacin: Key Points

- Improved efficacy and fewer side effects versus tolterodine

- Potential for CYP450 interactions with 3A4 inducers and inhibitors

- Favorable cost-effectiveness data

The newest anticholinergic drug in this class, fesoterodine was released in 2008, and is structurally very similar to tolterodine. Several studies have evaluated the efficacy of fesoterodine in comparison to tolterodine and have found fesoterodine to be superior in both efficacy and in patient-reported outcomes, such as amount of sleep and quality of social interaction. Another advantage of fesoterodine over tolterodine is that though both are metabolized to the active 5-hydroxymethyltolterodine (5-HMT), fesoterodine is done so by plasma esterases. Tolterodine is metabolized to 5-HMT in the liver by CYP2D6. 5-HMT is responsible for efficacy in the bladder as well as antimuscarinic activity systemically. In patients with pharmacogenomics variations in CYP2D6, the balance between toxicity and efficacy could be altered with tolterodine. Fesoterodine would not be affected by CYP2D6 polymorphisms [20] Unfortunately, side effects are no less common with fesoterodine versus tolterodine, with dry mouth being the most commonly reported reason for discontinuation [17].

Fesoterodine: Key Points

- Better efficacy and tolerability versus tolterodine

- Not subject to variable metabolism by CYP2D6 polymorphisms

- High incidence of side effects, especially dry mouth

Ultimately, anticholinergics are highly effective for treating overactive bladder and urge incontinence, but their side effects greatly limit their use. One systematic review looked at rates of anticholinergic therapy discontinuation in 12 -week clinical trials as well as in medical claims studies. In the clinical trials, discontinuation rates were as high as $31 \%$. In medical claims studies, discontinuation was even more prevalent, occurring in $43 \%$ to $83 \%$ of patients. Though not statistically significant, the review also demonstrated a trend in correlation between duration of therapy and discontinuation rates. These statistics further indicate the challenge of tolerability when using anticholinergics for urge incontinence, which usually requires long-term treatment [21].

\section{Desmopressin (DDAVP)}

Patients that suffer from overactive bladder symptoms often have significant nocturnal polyuria that interferes with effective sleep patterns. DDAVP is a synthetic replacement for vasopressin $(\mathrm{ADH})$ that reduces urine production at the level of the kidney [22]. It can be used with anticholinergic medications to help control nighttime urinary frequency. It should be used with caution in patients with hypertension, coronary artery disease, or congestive heart failure [23]. Since the medication may cause hyponatremia, patients should be advised to stop therapy if they experience vomiting or diarrhea. Also, it should be avoiding during hot weather or strenuous exercise that may cause excessive thirst [23].

\section{New Treatment Options}

In 2012, the FDA approved a new agent for overactive bladder and urge incontinence with a novel mechanism of action. Mirabegron is an agonist at the $\beta 3$ adrenergic receptor in the detrusor muscle. $\beta 3$ accounts for $95 \%$ of all $\beta$-adrenergic receptors in the bladder [24]. Binding at this receptor facilitates smooth muscle relaxation, thus reducing urge, frequency, and incontinence episodes without systemic anticholinergic side effects [25]. Early pharmacokinetic studies have shown multiple pathways active in the metabolism of mirabegron, including oxidation, glucuronidation, hepatic CYP3A4, hepatic CYP2D6 and alcohol dehydrogenase. While the potential for CYP450 interactions exists, the presence of multiple metabolic pathways reduces the likelihood of clinical significance of each. So far, large placebo-controlled studies have shown mirabegron to be very effective in reducing incontinence episodes, micturitions in 24 hours, and urgency episodes versus placebo and tolterodine [24]. In a most recent meta-analysis comparing mirabegron to other antimuscarinics, the results showed similar efficacy with incidence of dry mouth similar to placebo [26].

As a sympathomimetic, mirabegron has the potential to cause hypertension and reflex tachycardia. However, in a recent safety analysis including several randomized controlled trials, the rate of drug related hypertension was the same when comparing placebo with mirabegron [27]. Still, it is probably prudent to avoid this drug in patients with uncontrolled hypertension.

\section{Mirabegron: Key Points}

- Agonist at $\beta 3$ adrenergic receptors, which are concentrated in the bladder

- No anticholinergic side effects

- Multiple metabolic pathways reduce potential for drug interactions 
Citation: Matthews L, Shapiro R, Zaslau S. Pharmacologic Management of Overactive Bladder and Urge Incontinence: A Review of New and Old Treatment Options. J Androl Gynaecol. 2014;2(1): 4.

- Early studies have shown efficacy and favorable tolerability

- Avoid in patients with uncontrolled hypertension

\section{Conclusion}

Anticholinergic medications have been the primary medications used to treat urge urinary incontinence for decades. Though effective, they all produce side effects that continue to be bothersome for patients despite better muscarinic receptor selectivity. Overall, adherence and persistence to these medications remains very low, making management of this frustrating and embarrassing condition a challenge. Given its significant impact on quality of life, there has been a longstanding need for medications with better tolerability and new mechanisms of action. Mirabegron, a new agent with an alternative mechanism of action, seems to be both effective and well tolerated in early experience. Clinical experience and further comparative studies will be important in distinguishing the role of mirabegron in practice, but at this time it appears to be a promising new choice for patients unable to tolerate anticholinergic therapy.

\section{References}

1. Stewart WF, Van Rooyen JB, Cundiff GW, Abrams P, Herzog AR, et al (2003) Prevalence and burden of overactive bladder in the United States. World J Urol 20: 327-336.

2. Kavoussi LR, Novick AC, Partin AW, Peters CA (2011) Pathophysiology of incontinence and prolapsed. Campbell-Walsh Urology. Philadelphia, PA Elsevier Saunders.

3. Kavoussi LR, Novick AC, Partin AW, Peters CA (2011) Pelvic viscera bladder. Campbell-Walsh Urology. Philadelphia, PA: Elsevier Saunders.

4. Fry, $\mathrm{CH}$ (2013) The physiology and pharmacology of the urinary tract. Surgery 31: $329-336$

5. Krichevsky VP, Pagala MK, Vaydovsky I, Damer V, Wise GJ (1999) Function of M3 muscarinic receptors in the rat urinary bladder following partial outlet obstruction. J Urol 161: 1644-1650.

6. Abrams $\mathrm{P}$, Andersson $\mathrm{K}$ (2007) Muscarinic receptor antagonists for overactive bladder. BJU Int 100: 987-1006.

7. Gopal M, Haynes K, Bellamy S, Arya L (2008) Discontinuation rates of anticholinergic medications used for the treatment of lower urinary tract symptoms. Obstet Gynecol 112: 1311-1318.

8. Pagoria D, O'Connor RC, Guralnick ML (2011) Antimuscarinic drugs: review of the cognitive impact when used to treat overactive bladder in elderly patients. Curr Urol Rep 12: 351-357.

9. Goldenberg MM (1999) An extended-release formulation of oxybutynin chloride for the treatment of overactive urinary bladder. Clin Ther 21: 634642.

10. Pharmacologic features of the Oxytrol patch (2012) FDA Advisory Committee Briefing Document: Oxybutynin Transdermal System, 3.9 mg/day. Merck 1112.

11. Detrol LA: tolterodine extended release capsules (2012) Prescribing Information. Pharmacia and Upjohn, a division of Pfizer Inc. New York, NY.
12. Harvey MA, Baker K, Wells GA (2001) Tolterodine versus oxybutynin in the treatment of urge urinary incontinence: a meta-analysis. Am J Obstet Gynecol 185: 56-61

13. Zellner M, Madersbacher $\mathrm{H}$, Palmtag $\mathrm{H}$, Stohrer M, Bodeker $\mathrm{RH}$, et al. (2009) Trospium chloride and oxybutynin hydrochloride in a german study of adults with urinary urge incontinence: results of a 12-week, multicenter, randomized, double-blind, parallel-group, flexible-dose noninferiority trial. Clin Ther 31: 2519-2539.

14. Guay DR (2003) Clinical pharmacokinetics of drugs used to treat urge incontinence. Clin Pharmacokinet 42: 1243-1285.

15. Lam S, Hilas O (2007) Pharmacologic management of overactive bladder. Clin Interv Aging 2: 337-345.

16. Chughtai B, Levin R, De E (2008) Choice of antimuscarinic agents for overactive bladder in the older patient: a focus on darifenacin. Clin Interv Aging 3: 503-509

17. Drug Monographs. Micromedex Healthcare Series [Internet Database] Greenwood Village, CO: Thomson Healthcare. Updated periodically.

18. Chapple CR, Martine-Garcia R, Selvaggi L, Toozs-Hobson P, Warnack W, et al. (2005) A comparison of the efficacy and tolerability of solifenacin succinate and extended release tolterodine at treating overactive bladder syndrome; results of the STAR trial. Eur Urol 48: 464-470.

19. Ko Y, Malone DC, Armstrong EP (2006) Pharmacoeconomic evaluation of antimuscarinic agents for the treatment of overactive bladder. Pharmacotherapy 26: 1694-1702.

20. Herschorn S, Swift S, Guan Z, Carlsson M, Morrow JD, et al. (2009) Comparison of fesoterodine and tolterodine extended release for the treatment of overactive bladder: a head-to-head placebo-controlled trial. BJU Int 105: 58-66.

21. Sexton CC, Notte SM, Maroulis C, Dmochowski RR, Cardozo L, et al. (2011) Persistence and adherence in the treatment of overactive bladder syndrome with anticholinergic therapy: a systematic review of the literature. Int J Clin Pract 65: 567-585.

22. Weiss JP, Zinner NR, Klein BM, Nørgaard JP (2012) Desmopressin orally disintegrating tablet effectively reduces nocturia: results of a randomized, double-blind, placebo-controlled trial. Neurourol Urodyn 31: 441-447.

23. Weiss JP, et al. (2011) The evaluation and treatment of nocturia: a consensus statement. BJU Int 108: 6-21.

24. Sacco E, Bientinesi R (2012) Mirabegron: a review of recent data and its prospects in the management of overactive bladder. Ther Adv Urol 4: 315324.

25. Khullar V, Cambronero J, Angulo JC, Marianne W, Mary Beth B, et al. (2013) Efficacy of mirabegron in patients with and without prior antimuscarinic therapy for overactive bladder: a post hoc analysis of a randomized EuropeanAustralian phase 3 trial. BMC Urol 13: 45.

26. Nitti VW, Khullar V, van Kerrebroeck P, Herschorn S, Cambronero J, et al. (2013) Mirabegron for the treatment of overactive bladder: a prespecified pooled efficacy analysis and pooled safety analysis of three randomized double-blind, placebo-controlled, phase III studies. Int J Clin Pract 67: 619632.

27. Maman K, Aballea S, Nazir J, Desroziers K, Neine ME, et al. (2014) Comparative Efficacy and Safety of Medical Treatments for the Management of Overactive Bladder: A Systematic Literature Review and Mixed Treatment Comparison. Eur Urol 65: 755-765. 